\title{
Les S.H.S. en écoles d'ingénieurs, entre utilité pratique et formation culturelle
}

\author{
Michel Cotte ${ }^{\mathrm{a}}$ \\ Centre François Viète, Université de Nantes, France
}

\begin{abstract}
Résumé. L'article met en évidence la présence ancienne de deux pôles antagonistes dans les formations aux SHS ou Humanités dans un sens un peu plus large : leur utilité pratique au profit de l'ingénieur et son besoin de culture générale, notamment l'acquisition d'une vision stratégique de son action technique ; puis il examine à la lumière de cette opposition de traditions pédagogiques le contexte du développement de ces enseignements aujourd'hui.
\end{abstract}

\begin{abstract}
Contribution carries out two longtime trends producing antagonist poles in human and social sciences education, or Humanities in a bit broader sense: practical efficiency for the engineer versus its general cultural education especially to reach a strategic vision of his technical action; further it examines under the light of this opposition of pedagogical traditions the development of theses today teachings in context.
\end{abstract}

L'objectif de cette communication est de proposer dans sa première partie un regard historique assez général sur ce qu'il est aujourd'hui convenu d'appeler les sciences humaines et sociales (SHS) ou parfois les «Humanités » au sein des écoles d'ingénieurs françaises, pour désigner les enseignements non directement scientifiques ou technologiques. L'une des conclusions qui se dégage de cette première approche historique est la présence de deux traditions différentes du rapport des écoles à ces formations, suivant qu'elles se revendiquent comme des écoles généralistes ou comme des écoles spécialisées. La seconde partie de cette communication sera dédiée à examiner comment cette double tradition, pour nous clairement d'origine historique, se décline aujourd'hui dans les besoins de formation des ingénieurs en France. Cet article est suivi, dans la même publication, d'un second plus directement basé sur mon expérience professionnelle dans le domaine et qui tendra à illustrer le propos général et les questionnements apparus ici ${ }^{1}$.

\footnotetext{
a e-mail : michel.cotte@univ-nantes.fr

${ }^{1}$ La majeure partie de mes activités universitaires s'est exercée en école d'ingénieurs, en tant qu'historien des techniques. J'ai été successivement et parfois parallèlement, entre la fin des années 1980 et 2007, en charge de cours à l'ENTPE à Vaulx-en-Velin, à l'UT de Belfort-Montbéliard, à l'Ecole polytechnique de l'Université de Nantes, et à l'Ecole Centrale de Nantes. J'ai également dirigé le département des Humanités de l'UTBM et le département Homme Entreprise et Société à Polytech Nantes, expériences à la base du second article.

This is an Open Access article distributed under the terms of the Creative Commons Attribution License 4.0, which permits unrestricted use, distribution, and reproduction in any medium, provided the original work is properly cited.
} 
Notons que les termes de SHS ou d'Humanités en écoles d'ingénieurs sont des expressions récentes aux contours variables et souvent peu clairs. L'une des questions importantes qui accompagne cet article et le suivant est de contribuer à illustrer par des éclairages successifs cet ensemble notionnel qui constitue une succession de réalités, mais avec de notables différences de contenus, d'objectifs et de pratiques suivant les lieux et les époques. Ces termes sont compris et utilisés ici dans un sens large, c'est-à-dire ne faisant pas de référence exclusive à un ou à quelques enseignements spécialisés conformes à la définition universitaire classique des sciences humaines et sociales, mais à des ensembles variables d'une école à l'autre, d'un moment à un autre, au gré des besoins ressentis et des contextes. C'est l'une des questions que nous entendons aborder ici et éclairer : comment ces ensembles se sont-ils constitués et exprimés ? À quelles réalités institutionnelles et pédagogiques se rattachent-ils?

Nous utiliserons nous-même les deux termes au fil de ces articles, avec simplement une nuance entre eux : SHS renvoie plus volontiers vers des références académiques, Humanité vers un besoin de formation générale autre que de l'ordre des sciences exactes et des technologies au profit du jeune élève ingénieur. En situation de programme pédagogique d'une école donnée, ces disciplines ne revendiquent pas forcément ou peu le label des sciences humaines ou sociales, voire le rejettent au profit d'autres termes comme les « sciences de gestion », en référence à l'efficacité productive de l'entreprise, ou «sciences économiques » ou encore les «sciences de la communication » qui établissent un lien facile mais utile avec les langues, ou bien encore elles peuvent apparaître comme des segments limités ou même tronqués des habituels cursus académiques. Utiliser ces termes de SHS ou d'Humanité est un peu une facilité liée à ce que sont en réalité les grilles des enseignements non scientifiques, en écoles d'ingénieurs ; en d'autres termes une bannière de ralliement, un bout de secrétariat et un espace de bureaux parfois chèrement acquis. Il s'agit de définitions qui identifient un groupe d'enseignements, minoritaires et réputé comme «non scientifiques » n' apportant pas directement de compétence professionnelle dans la culture de beaucoup d'écoles, encore aujourd'hui. Nous sommes dans une enveloppe pragmatique et non dans une définition conceptuelle, qui prend d'ailleurs des noms divers et variés, plus ou moins poétiques, au gré des écoles : Humanités à l'UT de Belfort Montbéliard, CLE (Communication, langues, entreprise) à l'Ecole centrale de Nantes, HES (Homme, économie et société) à Polytech, etc., également en fonction de la présence ou non des langues en leur sein.

Je remercie les organisateurs de ce colloque de l'attention qu'ils portent à ces questions d'enseignement des sciences humaines en écoles d'ingénieurs, plus largement au sein des formations technologiques et scientifiques. Il y a sans doute eu de nombreuses réunions sectorielles ou de réseaux d'écoles ouvrant des discussions sur leurs pratiques pédagogiques et leurs objectifs, mais la volonté d'en faire un sujet de recherche à part entière est nouvelle, et il faut la saluer.

\section{Les origines historiques de la relation entre formations techniques et S.H.S.}

Les SHS en écoles d'ingénieurs sont une appellation générique assez large et même, pour être franc, plutôt confuse. Vu du terrain de la pratique, elles décrivent en premier lieu un périmètre pédagogique où la direction de l'Ecole place assez systématiquement tout ce qui ne dépend pas directement des enseignements scientifiques et technologiques, un peu comme le Tiers-Etat regroupait sous l'Ancien Régime tout ce qui n'était pas noblesse ou clergé ! Bien entendu, les métiers comme les compétences y sont variés et multiples, mais toujours au service du bien commun : la formation la meilleure possible des élèves/étudiants ingénieurs/techniciens, en un temps toujours limité, dans le respect du diplôme et du prestige de l'Ecole (aujourd'hui on dit « ranking », ou classement école, mais c'est la même chose). 


\subsection{Une question fort ancienne : L’Ecole des ponts et chaussées d'Ancien Régime}

Sous des vocables divers, les besoins d'enseignements complémentaires aux sciences et aux connaissances techniques sont fort anciens et leurs expressions historiques ont été assez diverses. Dès le départ des formations d'ingénieurs, notamment en France au XVIIIe siècle, des questions nombreuses se posent en prolongement des formations techniques et pour préparer à l'entrée dans les fonctions professionnelles. La technique n'est jamais là que pour elle-même, en tout cas pas pour le responsable d'un chantier ou d'une production, c'est-à-dire pour l'ingénieur mais aussi pour l'entrepreneur ou l'architecte ou le chef d'atelier. La technique s'inscrit toujours dans un contexte bien plus large, dans une société donnée à un moment donné, dans un système sociotechnique. Rappelons d'ailleurs qu'historiquement la fonction (d'ingénieur) précède l'organe (l'école) et que l'organe n'a pas toujours été nécessaire à la fonction. L'ingénieur civil britannique de la révolution industrielle n'a pas fréquenté d'école spécialisée, mais il vient de la pratique professionnelle dont il forme une élite à la fois technique et entrepreneuriale (Cotte 2005).

Reprenons avec Antoine Picon le parcours des élève à l'Ecole des ponts et chaussées sous l'Ancien Régime (Picon 1992), assez caractéristique de l'esprit de l'époque, même si cette école garde une forte spécificité. Les « degrés » accordés lors des concours ouverts périodiquement dans chaque discipline permettent à l'élève ingénieur de progresser dans la hiérarchie de l'école, vers la première classe, puis vers l'appel à une fonction au service ordinaire des Généralités et des Etats ou à un service spécialisé comme la construction d'un canal ou d'un port. Si les matières les plus nobles, c'est-à-dire les plus primées, sont bien les mathématiques et l'architecture, l'épreuve de «style » qui comprend des éléments esthétiques et d'écriture arrive immédiatement après, pratiquement au même niveau que la « coupe de pierres » ou l'épreuve de stéréotomie qui s'apparente à une géométrie dans l'espace à caractère pratique. Le dessin et ses variantes (cartographie, paysage, ornementation) occupent une place moins fortement récompensée, mais les archives de l'Ecole montrent que les élèves y accordent beaucoup de temps, pour des productions de haute qualité esthétique. La maîtrise de l'écriture, sur un plan graphique et pour la production de rapports faciles à lire ferment la liste (p. 118-120).

Quelles conclusions en tirer? La première est bien entendu l'importance relative mais forte des disciplines non directement techniques ; la seconde est certainement la continuité quasiment organique au XVIIIe siècle entre le technique et l'artistique, entre la qualité de conception et l'esthétique du résultat, entre le fond et la forme. Il n'y a finalement pas de frontière bien marquée entre ces différents domaines, et Antoine Picon a cette formule quand à la finalité éducative de cette première Ecole des ponts et chaussées : «former à un imaginaire analytique ». Cela se pratique par l'omniprésence $\mathrm{du}$ projet, qui est une approche globale de l'objet technique (route, pont, canal...) avec une décomposition - recomposition dans le champ de la pensée et par les outils de la mesure, du calcul, mais aussi du dessin et de l'écriture (p. 149-207).

Le recrutement à l'Ecole des ponts et chaussées est aussi très riche d'enseignements sur les pratiques sociales et les critères d'évaluation des postulants. L'examinateur de la Marine se déplace de port en port à la fin de l'Ancien Régime, pour sélectionner les futurs élèves, comme le fit un temps Monge (Pairault François 2000). Des examinateurs successifs qui par souci d'équité et de justice entre les candidats en arrivent à inventer la colle de math... Avant même cette invention « démocratique », les pratiques de Perronet, le fondateur et inamovible directeur de l'Ecole des ponts et chaussés sous l'Ancien Régime, retiennent l'attention ${ }^{2}$.

A l'entrée de l'Ecole des ponts et chaussée d'Ancien Régime, il n'y a pas à proprement parler de concours, mais une pratique systématique de la lettre de recommandation comme première sélection, puis un entretien oral, aussi redouté que déterminant, avec le directeur en personne. A travers ces filtres, il faut tout d'abord attester par la recommandation d'un niveau d'éducation scientifique, artistique et

2 Perronet dirigea l'Ecole de sa création en 1747 à la Révolution. 
littéraire suffisant, ainsi que d'une origine sociale satisfaisante pour les critères culturels de l'époque. L'entretien avec Perronet et ses assistants permettait ensuite de le vérifier. Il permettait d'apprécier d'autres éléments à leurs yeux essentiels au bon exercice du métier d'ingénieur : la facilité d'élocution, le maintien personnel et la capacité à paraître pour représenter convenablement le corps devant les élites et l'aristocratie des généralités, une taille et une robustesse suffisante pour vivre au grand air et s'imposer aux ouvriers, etc. De fait, les élèves des Ponts et Chaussées de cette période appartiennent majoritairement à un milieu social bien défini : celui d'une élite bourgeoise parisienne et des grandes villes de province, dont la famille se consacre soit aux offices publics de l'administration de l'Etat soit au négoce et aux entreprises. Un niveau minimum de fortune personnelle est requis. Par opposition, à la même époque, l'Ecole du génie de Mézières recrute surtout les enfants doués de la petite noblesse provinciale et rurale, d'un niveau de revenu pas forcément très élevé (Picon Antoine, op. cité, p. 90-103).

A l'évidence, les critères du recrutement débordent largement dans le domaine de l'éducation générale, et pas seulement celui d'une formation scientifique et professionnelle préalable. Mais Perronet juge plus qu'une éducation : il valide la maîtrise d'une culture comme signe d'appartenance à une classe sociale, elle-même garante d'une d'efficacité de l'action au sein de la société de l'époque, tant vis-à-vis $\mathrm{du}$ « haut » (les décideurs) que du « bas » (les ouvriers). Bien entendu, l'ensemble du cursus à l'Ecole permet de vérifier si l'impression de départ est bonne, parallèlement à la progression des degrés et des classes de l'élève. S'il y a eu erreur de jugement ou inflexion négative au cours de la scolarité, il n'y aura tout simplement pas d'appel à une fonction au service du royaume !

\subsection{La formation des dirigeants industriels mulhousiens au tournant XVIIle - XIXe siècle}

Les stratégies des familles patronales éclairent également sur les pratiques culturelles de la formation de leurs jeunes destinés à diriger l'entreprise, notamment au moment où l'idée de révolution industrielle devient une réalité, et cela indépendamment de la notion même d'école. À la suite de Louis Bergeron (Bergeron 1992) et de Florence Ott (0tt 1999), je me suis intéressé à la formation des cadres et des dirigeants des entreprises mulhousiennes à la fin du XVIIIe siècle et au début du suivant, formant un exemple bien documenté qui illustre des pratiques valable à l'échelle de l'Europe occidentale et centrale, à cette époque. C'est un moment décisif de l'histoire de cette ville, par un décollage précoce et bien coordonné de son industrie déjà diversifiée : le tissage des étoffes de coton, la chimie pour les colorants, la mécanique pour le machinisme textile et l'usage de l'énergie vapeur. Le milieu patronal y est bien organisé, créant rapidement des institutions collectives efficaces comme la Société industrielle de Mulhouse (1825-1826), puis ses propres écoles techniques. La formation des premières générations des dirigeants industriels mulhousiens est intéressante à plus d'un titre. Il s'agit d'une formation plurielle dans ses temporalités comme dans ses objectifs, se basant sur les grandes traditions culturelles du négoce, notamment au sein du Monde rhénan et de la Suisse voisine, mais que l'on retrouve avec des variantes dans toute l'Europe, comme un fond culturel commun qui s'est développé dès la Renaissance (Cotte 2005).

Après une éducation familiale puis dans les écoles locales des pasteurs calvinistes, la règle est de partir dans un collège, généralement en Suisse. Ceux-ci dispensent une éducation moderne réputée, tant pour son enseignement (sciences, langues vivantes) que pour sa pédagogie ouverte et vivante. L'apprentissage d'un métier est aussi dans les habitudes. Il est parfois précoce, mais il intervient souvent après le collège, en général à l'étranger, chez un collègue anciennement connu, du même métier que la famille ou dans un domaine proche, puis, pour la génération suivante, directement à l'école des entreprises britanniques.

On retrouve là les pratiques du « grand tour» des jeunes élites négociantes, parties pendant quelques mois ou années de leur jeunesse, à la découverte tant culturelle que professionnelle de régions européennes plus ou moins lointaines (Hibbert, 1987). Très vite ensuite, un séjour complémentaire est 
Les sciences humaines dans les parcours scientifiques et techniques professionnalisants

\begin{tabular}{|c|c|c|c|}
\hline $\begin{array}{l}\text { Nom, } \\
\text { Spécialité ; ville si autre que } \\
\text { Mulhouse }\end{array}$ & $\begin{array}{c}\text { Etudes scolaires, } \\
\text { institutions ; Apprentissages }\end{array}$ & $\begin{array}{c}\text { Voyages et séjours de } \\
\text { jeunesse }\end{array}$ & $\begin{array}{l}\text { Formation scientifique, } \\
\text { école d'ingénieur }\end{array}$ \\
\hline $\begin{array}{c}\text { Jean Zuber père, (1772- ?) } \\
\text { papiers peints }\end{array}$ & $\begin{array}{l}\text { Ecoles publiques et privées } \\
\text { locales ; Négoce }\end{array}$ & $\begin{array}{l}\text { Italie ( } 2 \text { fois), Espagne, } \\
\text { Allemagne }\end{array}$ & \\
\hline $\begin{array}{l}\text { Nicolas Koechlin père (1781- } \\
1852) \text {, industrie, ch. de fer }\end{array}$ & Négoce & Hambourg, Hollande & \\
\hline $\begin{array}{c}\text { Daniel Koechlin- Schouch } \\
(1785-1871)\end{array}$ & Maroquinerie & & Fourcroy, chimiste, Paris \\
\hline $\begin{array}{l}\text { André Koechlin (vers 1790- } \\
1837 \text { ) }\end{array}$ & Ecole de Lenzburg $(\mathrm{CH})$ & & Ecole polytechnique \\
\hline $\begin{array}{c}\text { Joseph Koechlin- } \\
\text { Schlumberger (1796- 1863) }\end{array}$ & Ecole d'Yverdon $(\mathrm{CH})$ & & $\begin{array}{l}\text { Etudie la chimie à } \\
\text { Mulhouse }\end{array}$ \\
\hline $\begin{array}{c}\text { Jean Zuber fils (1799-1853) } \\
\text { papiers peints }\end{array}$ & & & Etudie la chimie à Paris \\
\hline $\begin{array}{l}\text { Daniel Dollfus-Ausset (1797- } \\
\text { 1870) coloriste et chimiste }\end{array}$ & Ecole d'Aarau $(\mathrm{CH})$ & & Chevreul, chimiste, Paris \\
\hline $\begin{array}{l}\text { Laurent Schwartz (1802- } \\
\text { 1885) coloriste et chimiste }\end{array}$ & Etudes à Nancy & & Thénard, chimiste, Paris \\
\hline Matthieu Hofer (1803-1832) & Ecole à Hofwyl $(\mathrm{CH})$ & $\begin{array}{l}\text { Apprenti à Zurich, } \\
\text { Constantinople }\end{array}$ & $\begin{array}{l}\text { Chimie et mécanique à } \\
\text { Paris }\end{array}$ \\
\hline Emile Dollfus (1805-1858) & $\begin{array}{l}\text { Suisse : St-Gall, Hofwyl, } \\
\text { Lausanne ; Mécanique }\end{array}$ & Bruxelles, Manchester & Conservatoire, Paris \\
\hline $\begin{array}{l}\text { Jules Albert Schlumberger } \\
\text { (vers 1805-) ingénieur méca. }\end{array}$ & & Angleterre & Conservatoire, Paris \\
\hline $\begin{array}{c}\text { J.J. Vetter (1805- ) Ingénieur } \\
\text { mécanicien }\end{array}$ & Mécanique & Angleterre & $\begin{array}{l}\text { Pharmacie, Grünstadt (All) } \\
\text { Ecole des A.M. de Châlons }\end{array}$ \\
\hline $\begin{array}{c}\text { Charles Mertzdorff (1818-83) } \\
\text { Teinturier au Vieux-Thann }\end{array}$ & $\begin{array}{l}\text { Ecole locale, séminaire, } \\
\text { Ecole Töppfer à Genève }\end{array}$ & Manchester (2 ans) & $\begin{array}{l}\text { Labo de Liebig, chimiste } \\
\text { (All) }\end{array}$ \\
\hline $\begin{array}{c}\text { Gustave Steinheil (1818- } \\
\text { 1906) fabricant textile Bas- } \\
\text { Rhin }\end{array}$ & $\begin{array}{l}\text { Gymnasium protestant de } \\
\text { Strasbourg, mécanique }\end{array}$ & $\begin{array}{l}\text { Employé de commerce à } \\
\text { Rouen, Paris }\end{array}$ & Ecole de commerce Liepzig \\
\hline $\begin{array}{l}\text { Henri-Albert Koechlin } \\
(1823-1859)\end{array}$ & Apprenti coloriste à Cernay & Glasgow & $\begin{array}{l}\text { Chimie à Mulhouse; labo de } \\
\text { Persoz, Strasbourg; labo de } \\
\text { Liebig (All.) }\end{array}$ \\
\hline
\end{tabular}

Figure 1. Quelques trajectoires de formations des patrons de Mulhouse et d'Alsace au début du XIXe siècle (Cotte 2005, p. 102).

effectué dans un laboratoire, notamment pour les futurs coloristes-chimistes au sommet de la hiérarchie professionnelle mulhousienne de cette époque. Pour la seconde génération, la fréquentation des écoles d'ingénieurs ou assimilées apparaît, Polytechnique à Paris, mais surtout les Arts et Métiers, en lien avec la mécanique des machines.

Dans ce type d'éducation se côtoient en premier lieu une culture générale ouverte au monde moderne et à l'apprentissage de savoir-faire professionnels précis. Puis l'ensemble évolue très vite vers l'acquisition des connaissances scientifiques, en privilégiant le côté appliqué et pratique (laboratoires de chimie, Arts et Métiers). L'un des intérêts évidents de tels parcours est une bonne préparation des individus, d'une part aux affaires dans un cadre spontanément international, facilité par la position de carrefour de Mulhouse, d'autre part à une connaissance des techniques étrangères et à leur adaptation au contexte local à leur retour. Il faut noter et même souligner que dans cette forme d'éducation qui prélude et accompagne l'industrialisation, les savoir-faire techniques et la science viennent se greffer sur un modèle existant, celui du négoce international, dont la base éducative restait la culture générale au sens de la connaissance du monde, de la pratique des langues étrangères, de la curiosité en général et plus particulièrement de celle des affaires et de l'argent. 
Dans les deux exemples précoces de formation à l'initiative technique et à la responsabilité entrepreneuriale que nous venons d'évoquer, l'imbrication des données culturelles avec celles des connaissances et des savoir-faire est une forme d'évidence, qu'il semble bon de rappeler. C'est en effet un point de départ qui va s'affadir et même parfois se perdre au cours du XIXe siècle, notamment dans le cadre du développement des écoles d'ingénieurs en France. Gardons à l'esprit cette idée d'un besoin de formation autre que scientifique ou technologique, mais en symbiose avec celui-ci. C'est un gage de qualité comme de capacité personnelle indispensable pour donner du sens et de l'efficacité aux compétences purement techniques, mais c'est aussi un accompagnement polymorphe aux définitions des plus variables, en fonction du contexte d'un marché économique donné à un moment donné.

\subsection{Vers deux modèles de formation des ingénieurs au XIXe siècle : le spécialiste et le généraliste}

Nous avons examiné deux exemples fondateurs, à notre point de vue, au tournant du XVIIIe siècle vers le suivant. Sont-ils généralisables et quelles vont-être les tendances aux formations non scientifiques dans le mouvement de création des écoles d'ingénieurs qui se généralise au cours du XIXe siècle ? La première difficulté sérieuse à se poser dans la formation des ingénieurs, notamment dans le modèle français de l'école, n'est pas celle du rapport entre culture et technicité, mais bien plutôt celle des relations et de la place des sciences, en tant que corpus de connaissances en rapide expansion, face à la pratique, face au savoir-faire et à l'expérimentation. L'Ecole du génie de Mézières a sans aucun doute été pionnière dans ce domaine, faisant se côtoyer une « école de théorie » et une « école de pratique » (Belhoste 1997).

La première version de l'Ecole polytechnique de la Révolution (1794-96) avait une ambition similaire, reprise de Mézières, mais qui fit long feu pour différentes raisons, tant organisationnelles qu'en raison du rapport de force entre l'Ecole et les corps d'ingénieurs constitués en services d'Etat (Belhoste 1994). Ceux-ci, d'ordre militaire ou civil, furent des agents déterminants de la réussite des entreprises de conquête militaire puis de contrôle et de développement territorial sous la Révolution puis l'Empire. L'une de leurs revendications corporatistes était de garder leurs écoles propres, justement dédiées à la pratique professionnelle, aux campagnes de terrain et à l'apprentissage des réalités du métier. Le concept d'une répartition des tâches s'ensuivit : Polytechnique comme tronc commun d'une formation initiale scientifique généraliste et de haut niveau, puis le choix du corps et de son école d'application : le Génie militaire, les Mines, les Ponts et Chaussées, l'Artillerie, etc. Nous ne sortons de notre sujet qu'en apparence, car les Humanités, au sens large et polymorphe que nous venons de voir, disparaissent à ce moment-là des priorités de la formation des ingénieurs. Ce phénomène d'effacement est provoqué tout aussi bien par le nouveau cadre chronologique de la formation polytechnicienne, qui les renvoie implicitement aux écoles d'application où elles restent marginales, que par les bouleversements sociaux et culturels d'une période passionnée d'efficacité rationnelle. Le rapide développement des sciences est directement enseigné par les nombreux savants qui interviennent au sein de la formation polytechnicienne, comme une priorité pédagogique absolue, avec une attention particulière accordée aux sciences exactes, les mathématiques analytiques et la physique théorique notamment. Le sentiment d'avoir trouvé le langage universel de la science, et qu'il peut à lui seul prévoir la technique (Navier 1823), ouvre vers une forme de scientisme éducatif, au sens d'une connaissance prédictive qui prétend transcender son contexte et donc s'affranchir de la culture. La science est universelle en elle-même et elle dépasse les contingences qui deviennent secondes, voire qui constituent des freins à la rationalisation. La Science se suffit alors à elle-même, alpha et oméga du développement technologique et du progrès humain, ce qu'exprime notamment l'idéologie positiviste d'Auguste Comte.

Même si le modèle pédagogique polytechnicien est appelé à un avenir prestigieux et à une diffusion internationale importante, il ne concerne qu'une frange bien précise de la société française du XIXe siècle : ses élites militaires et administratives, en particulier ses meilleurs professionnels des sciences exactes. D'autres écoles, d'autres lieux de formation apparaissent cependant dès la fin du XVIIIe 
siècle, en lien plus directement avec les besoins des manufactures puis de l'industrie naissante : les Ecoles d'arts et métiers de Chalons, d'Angers et d'Aix-en-Provence avant 1850 ; le Conservatoire des arts-et-métiers à Paris durant la Révolution. Il s'agit-là de lieux où l'on enseigne essentiellement des capacités techniques et un savoir-faire relativement spécialisé, en direction de l'usage et de la fabrication des premières machines mécaniques. On peut ranger dans cette catégorie des écoles techniques spécialisées : l'école de chimie de Mulhouse (1822) ou celle de la Martinière à Lyon (1829). On y rencontre des formes importantes d'enseignements complémentaires aux formations techniques et de savoir-faire : le dessin sous toutes ses formes, l'écriture, en lien direct avec des finalités professionnelles. Il s'agit d'écoles qui ne revendiqueront que plus tard le titre d'école d'ingénieur, à la fin du XIXe siècle, voire pas du tout. Il s'agira alors d'un ingénieur ou d'un technicien hautement spécialisé en mécanique, en chimie des colorants, puis en électricité, en électronique, en informatique aujourd'hui, etc. Elles expriment le besoin d'une compétence professionnelle clairement définie et aux limites bien reconnues, pour une responsabilité industrielle précise. La spécialité est ici un gage d'efficacité et de compétence.

Deux types d'ingénieurs apparaissent donc au cours du XIXe siècle, en France, de manière à peu près parallèle : le généraliste et le spécialiste. Le modèle de l'ingénieur généraliste est un peu plus complexe à appréhender, car sa définition est moins claire que celle du spécialiste. Longtemps, en France, le terme « ingénieur » fut réservé aux seuls ingénieurs des corps d'Etat, tous passés par le moule polytechnicien à partir de la Révolution. Par la diversité de leurs fonctions et la généralité de leurs responsabilités, ils peuvent apparaître comme les inventeurs de l'ingénieur généraliste, du moins en France. Mais nous avons vu que leur formation faisait une double part, de généraliste certes, mais surtout des sciences, et de spécialiste par le choix d'un corps et donc de son école d'application. La formation qui se réclame le plus clairement d'une approche généraliste des techniques est l'Ecole centrale des arts et manufactures (1829), en se proposant de former les cadres de l'industrie et les futurs dirigeants que le monde industriel réclame pour développer ses vastes projets (Cotte 2007).

Dans le contexte de la formation de l'ingénieur généraliste au XIXe siècle, future élite de la nation ou futur capitaine d'industrie, la place des Humanités gagne, ou plutôt regagne, un rang plus notable : les valeurs culturelles et sociales de la bourgeoisie du XIXe siècle, en plein épanouissement, doivent avoir leur place dans le profil de l'ingénieur devenu l'une des icones de sa réussite et du siècle. Le plus souvent, il s'agit d'un bagage social et culturel d'origine familiale, formé lors de l'éducation secondaire, et qui est considéré comme un acquis à l'entrée de l'école plutôt qu'un cursus d'enseignement. Ce qui joue un rôle important, c'est par exemple le stage, la campagne de terrain (Mines, P \& C), que l'on doit accompagner d'un rapport de bonne tenue scientifique et technique, mais aussi littéraire ${ }^{3}$. Les impératifs financiers et comptables tendent aussi à se faire une place en propre, jugés comme indispensables au bagage du futur ingénieur.

À notre point de vue, le XIXe siècle définit et justifie les fondements des enseignements non scientifiques au sein des écoles via l'apparition de deux traditions opposées, produites par leurs pratiques pédagogiques en lien étroit avec la définition culturelle plus large du rôle que ces écoles entendent jouer dans la société française. Les deux pôles qui vont gouverner les formations non scientifiques en écoles sont en place : d'abord les enseignements directement utiles en prolongement des formations technologiques, avec le souci d'en augmenter l'efficacité, et ensuite, mais seulement dans les plus grandes écoles à caractère généraliste, un certain souci de culture générale et de vision stratégique de l'entreprise. Ils marquent durablement et jusqu'à aujourd'hui le paysage de la formation des ingénieurs en France, et par influence culturelle dans bon nombre d'autres pays. C'est ce besoin de formation actuel que nous entendons maintenant examiner, à la lumière de la dichotomie "spécialiste - généraliste » que nous venons d'établir. L'apport historique joue pour nous le rôle d'établissement d'une grille d'observation et de compréhension des phénomènes à l'œuvre aujourd'hui. Avant même d'être une

\footnotetext{
${ }^{3}$ Les meilleurs de ces rapports sont publiés dans les Annales des Mines et les Annales des Ponts et Chaussées, dont c'est l'un des rôles, et qui amorcent une notoriété scientifique pour l'auteur.
} 
problématisation de la question des SHS en écoles d'ingénieur, cela a été pour nous un facteur important de réflexion, avec les élèves ingénieurs eux-mêmes, via les cours d'histoire des ingénieurs.

\section{La définition aujourd'hui des besoins de formation en S.H.S.}

\subsection{La traduction contemporaine des deux pôles du spécialiste et du généraliste}

Un double modèle de formation s'est donc progressivement développé jusqu'à aujourd'hui, et on le retrouve aussi hors de France, dans les pays latins, en Russie, etc. Au sein d'une école donnée, les dimensions du généraliste et du spécialiste se marient toujours à des degrés divers, ne serait-ce que par les enseignements scientifiques généraux d'un côté et les enseignements techniques et de spécialités qui existent toujours de l'autre à un degré ou un autre. La formation à une spécialité peut aussi évoluer vers des formes de polyvalence technique. Tout est une question de degré, de temps consacré dans les plannings pédagogiques, de qualité et de style des enseignants aussi. Chaque école a donc un espace pour créer son image de marque, affirmer une ambition et cibler un marché de l'emploi, enfin pour produire une image la plus personnalisée possible et la plus flatteuse d'elle-même.

Identifions rapidement quelques exemples caractéristiques de ces deux grandes tendances. Les écoles à dominante "spécialiste »s'ancrent dans le réseau ancien des écoles des Arts et Métiers, les Gadzarts à destination de l'industrie. Elles furent des pourvoyeuses historiques d'ingénieurs mécaniciens pour la France. Il y a aussi les écoles des mines de province ${ }^{4}$ (Saint-Etienne, Alès, Douai) ou encore l'ENTPE 5 aujourd'hui en banlieue lyonnaise. A la fin du XIXe siècle, la création de Supélec vint apporter une réponse de formation spécialisée de haut niveau, qui cherchait à combler un retard technique, notamment sur les ingénieurs électriciens allemands nombreux et biens formés. Plus récemment, les anciennes ENSI de l'après-guerre, les INSA des trente glorieuses, les «petites écoles » issues des milieux professionnels ${ }^{6}$, puis à la fin du XXe siècle les nouveaux venus comme le réseau des Polytech (écoles d'ingénieurs en université classique) et d'innombrables écoles d'informatique revendiquent à des degrés divers leur vocation à former des ingénieurs spécialisés. En d'autres termes, elles affichent (ou affichèrent) une dimension «métier» bien identifiée et directement exploitable par un industriel ou un service public à caractère technique.

Les écoles à dominante généraliste sont en premier lieu les Grandes Ecoles historiques, soit Polytechnique et ses écoles d'applications issues de la Révolution et de l'Empire, mais non sans ambiguité. En fait, le haut niveau scientifique est le ciment généraliste, garant de la polyvalence, mais les sciences humaines et sociales peuvent assez spontanément fournir une seconde couche de " généralité », cette fois par la culture. Il faut ajouter l'Ecole centrale parisienne que l'on peut considérer comme la véritable fondatrice du concept de l'école généraliste en regard du monde de l'entreprise privée, en indiquant clairement son but de formation des dirigeants des nouvelles entreprises industrielles. Elle est historiquement un exemple de formation par la pluridisciplinarité technique qui, par ailleurs, s'est rapidement appuyée sur le modèle scientifique exigeant de l'X. «Centrale » s'est illustrée par la réussite remarquable de ses ingénieurs dans la direction des entreprises industrielles françaises, depuis le milieu du XIXe siècle (Barjot 2008).

Aujourd'hui, on observe une mise en réseaux d'écoles d'origines différentes et des évolutions. Le réseau des Mines affiche, dans la lignée de Mines Paris, Grande Ecole s'il en est, une vocation généraliste d'autant plus importante que l'exploitation minière en tant qu' activité nationale a totalement

\footnotetext{
${ }^{4}$ Elles fournissent des cadres intermédiaires aux services publics régionaux, mais surtout les responsables d'exploitations des entreprises concessionnaires des mines.

${ }^{5}$ L'Ecole nationale des Travaux Publics de l'Etat formait initialement les conducteurs de travaux, assistants et suppléants des ingénieurs titulaires X-Ponts ; ils ont ensuite accédé au titre officiel d'ingénieur de l'Etat. Son équivalent privé fut l'ESTP.

${ }^{6}$ Par exemples des écoles crées par les Chambres de commerce pour répondre à des besoins spécifiques de certains bassins d'emploi.
} 
disparue ; mais il est constitué en grande partie par les anciennes écoles provinciales autrefois très spécialisées. Le réseau Centrale a une histoire un peu similaire, appuyé sur des écoles régionales au passé parfois complexe. Il y a donc des histoires d'écoles et des évolutions importantes sur l'échiquier de la formation des ingénieurs, comme l'ancienne école de l'EPO à Nantes, crée au lendemain de la Grande Guerre, devenue ENSI de mécanique à la Libération, puis l'une des écoles typiques du réseau centralien actuel (Hervouët et Fonteneau 2009). Il faut également citer un réseau nouveau venu, les universités de technologie créées à la suite de l'UT Compiègne, se réclamant ouvertement d'une formation généraliste de l'ingénieur, notamment par un rôle important attribué aux SHS dès leur fondation ${ }^{7}$. Les grandes ambitions de développement affichées par les UT, un peu comme pour les INSA des années 60-70, ont cependant connu un coup d'arrêt, pour se limiter aux trois écoles des années 90 : Compiègne, Belfort et Troyes (Lamard et Lequin 2005).

Pour les écoles à la spécialité revendiquée (ou longtemps revendiquée), c'était d'abord une reconnaissance de la compétence professionnelle comme manière de s'affirmer sur le marché de l'emploi des cadres. Les SHS y tenaient par définition un rôle fonctionnel relativement précis mais étroit, en complément direct de la formation technique spécialisée. Ces écoles ont souvent suivi une évolution par l'élargissement des compétences métiers autour d'un noyau dur initial (la mécanique au XIXe siècle, l'électricité début XXe, l'électronique dans l'Après-guerre, l'informatique à la fin du $\mathrm{XXe} . .$.$) , ce qui les a amenées à diversifier leur offre de spécialités ou bien à les faire évoluer vers une$ plus grande pluridisciplinarité, porte ouverte vers une dose de formation généraliste. Les formations aux spécialités se régénèrent souvent par la recherche de niches métiers au moment de leur apparition ou même en anticipation. S'il y a eu de belles diversifications du côté de l'informatique, d'autres secteurs ont souffert ou bien ont mis du temps à s'imposer comme les ingénieurs en biotechnologie ou dans les métiers de l'environnement.

L'une des conséquences pour l'organisation des écoles a été un mouvement de croissance par départementalisation, encouragé par le principe de la reconnaissance du diplôme d'ingénieur en correspondance avec une branche professionnelle bien identifiée par la Commission du titre de l'ingénieur (CTI). Un fédéralisme rassembleur mais producteur de spécialités s'est alors mis en place, avec parfois le regroupement de petites entités antérieures fort différentes ${ }^{8}$, simplement pour atteindre une masse critique en termes de lisibilité et d'attractivité. La conséquence institutionnelle est souvent, mais pas toujours, une faible intervention des directions d'écoles dans le champ pédagogique, le véritable pouvoir se situant dans les départements qui préparent les maquettes d'enseignement, ensuite validées par la CTI plus que par l'Ecole elle-même. L'étape du conseil des études n'est alors qu'une harmonisation des présentations et la recherche d'un langage commun souvent puisé dans les textes même de la CTI.

\subsection{L'impact variable de ces traditions en termes de formation par les SHS}

Dans le cadre de d'école de spécialistes par accolement de départements (l'école «tuyaux d'orgues ») ou même d'écoles de spécialités aux branches mieux structurées entre elles, le rôle des SHS, dans le sens de l'introduction de disciplines assez bien identifiées, ou d'Humanités dans un sens plus large, a longtemps été jugé comme secondaire. Il est d'abord perçu comme un complément utile et parfois très basique des formations techniques. Savoir faire un CV était le $1^{\text {er }}$ besoin cité par les directeurs de départements de Polytech Nantes, lors de la refonte de la maquette Homme Economie Société (HES) au milieu des années 2000 ! On peut y ajouter : avoir des notions de base en gestion, pratiquer l'anglais au niveau minimal du TOEIC, limiter le nombre de fautes de français dans un rapport, etc.

\footnotetext{
7 Voir dans ce recueil l'article de Lenay et collaborateurs.

${ }^{8}$ Par exemple la fusion - absorption de l'ENI de Belfort par l'IPSE pour donner l’UT Belfort Montbéliard.
} 
Au cran immédiatement supérieur, pour des écoles affichant un minimum de formation généraliste dans leur programme, les SHS ou les Humanités, sont perçues comme susceptibles de fournir des « plus» d'efficacité sur des points spécifiques, comme la gestion de production (taylorisme, toyotisme...), l'anglais professionnel, la gestion des équipes, des rudiments de comptabilité et de négociation commerciale, etc. Une école de ce type dispose généralement d'une équipe pédagogique interne relativement réduite pour les SHS/Humanités, et elle a recours à de nombreux intervenants extérieurs. Elle privilégie les formations courtes, par des spécialistes aux compétences appliquées bien identifiées : par exemple un intervenants en comptabilité, en gestion des RH, en qualité - sécurité, un PRAG d'anglais, un spécialiste du français pour étudiants étrangers, etc. L'expérience en entreprise est souvent un pré-requis de ces intervenants, plus que leurs qualifications universitaires. En d'autres termes, il s'agit d'une gestion parcimonieuse des SHS dont l'objectif affiché est un complément efficace des formations technologiques. L'école embauche donc un nombre important de vacataires de niveau moyen, parfois de vrais-faux professionnels spécialistes de la prestation en écoles, sur un créneau porteur et facile à vendre, comme un bloc d'heures bien identifiable dans la grille horaire. Ce type d'école a en outre et presque toujours une prédilection pour la valorisation des sports collectifs, les projets à enjeux en petits groupes comme les éco-marathons automobiles, l'humanitaire technique, etc. Les stages à l'étranger, autrefois assez limités en nombre se sont aujourd'hui généralisés, ainsi que la recherche du double diplôme pour les écoles les plus ouvertes à l'international. Il s'agit d'une image dynamique à promouvoir auprès des candidats potentiels à l'entrée dans l'école, tout comme la participation active des élèves ingénieurs aux salons d'étudiants pour favoriser le recrutement. Tout cela forme souvent le package «SHS ou Humanités ou autre chose » tel que vu par les directions de départements d'ingénieurs spécialistes. On conviendra de son côté protéiforme mal identifié qui peut dérouter un jeune collègue issu du sérail académique des «vraies SHS » telles qu'elles sont aujourd'hui définies, mais pas forcément depuis très longtemps... Dans ce type d'école, la gestion des espaces horaires SHS/Humanités s'apparente à la gestion d'un ensemble compliqué de morceaux de cours et d'interventions périphériques, à intégrer dans les espaces laissés vides par les enseignements technologiques et scientifiques.

Pour le second groupe des écoles à caractère généraliste revendiqué, l'objectif d'un accès à des fonctions élargies de responsabilités industrielles ou administratives favorise une vision plus globale et plus culturelle de la formation aux SHS. Les objectifs affichés sont tout de suite plus ambitieux, et ils s'affirment au niveau de l'Ecole dans son ensemble. C'est même une tendance naturelle des Grandes Ecoles qui ont compris, parfois depuis longtemps comme Mines Paris, l'importance de SHS de haut niveau en écoles d'ingénieurs, pour accéder à des visions stratégiques de l'industrie et de ses changements. Là, la création d'un laboratoire comme le Centre de sociologie de l'innovation', la tenue de séminaires de formation de qualité, des publications accompagnent le mouvement pédagogique et lui donne une consistance scientifique. Le réseau des UT a également eu la volonté de créer une recherche originale, à l'interface des techniques et des sciences humaines et sociales, comme par exemple l'étude des contextes historiques et territoriaux du fait industriel par l'équipe pluridisciplinaire de RECITS à l'UT Belfort ${ }^{10}$, l'approfondissement du design industriel à l'UT Compiègne, le rôle de l'ergonomie dans plusieurs écoles, etc. Par ailleurs, sur un plan organisationnel, ces écoles ont une tradition forte de direction pédagogique intégrée, et les conseils de perfectionnement ou des études y jouent plus volontiers un rôle moteur et créateur que dans les écoles de spécialités. Les Humanités y sont perçues comme un véritable département, transversal de l'école, et non comme une sorte de service auxiliaire des départements technologiques.

\footnotetext{
${ }^{9}$ Créé en 1967, il s'est notamment développé dans les années 1980 avec M. Callon et B. Latour.

10 Créé en 1998, dans le cadre du département des Humanité par une équipe d'historiens interne à l'UT : Belot R., Lamard P. et l'auteur de cet article.
} 
Dans ces écoles à dimension généraliste, le besoin de préparer les élèves à l'exercice de responsabilités élargies, de la promotion de l'innovation en interne via la surveillance des concurrents (R\&D, service brevets, benchmarking...) à la prise en compte des fonctions commerciales et de marketing technique (clients, fournisseurs, concurrents, partenaires étrangers... ) jouent à plein en faveur d'enseignements d'économie, de sociologie, d'histoire, etc. Citons l'exemple du droit, un domaine qui entretient de nombreux rapports avec l'entreprise, dans des domaines très divers (droit de l'entreprise, droit du travail, propriété intellectuelle, droit commercial international, marchés publics, etc.) : il s'agit de formations brèves et synthétiques visant à une initiation, mais de bon niveau. Il ne s'agit pas de former des juristes au sens plein du terme, mais d'apporter une culture juridique suffisante pour identifier rapidement un problème dans l'entreprise, et savoir trouver le bon interlocuteur pour le traiter convenablement. Ces écoles ont le souci des langues autres que l'anglais et une sensibilité à l'inter-culturalité par l'introduction aux civilisations des futurs partenaires étranger (monde anglosaxon, BRICS, etc.), également à la géopolitique et à l'histoire économique internationale, ou encore à des introductions de bon niveau scientifique à la gestion des ressources humaines. La tendance est là souvent de considérer les SHS comme un plus de formation, comme une valeur ajoutée par l'Ecole. Une sensibilité à la culture générale de l'ingénieur, à une forme d'ouverture d'esprit à l'international existe assez spontanément.

Ces écoles acceptent plus facilement la mise à disposition de moyens pédagogiques (horaires, locaux, formation d'une équipe pédagogique interne conséquente, bibliothèque, etc.) avec un souci d'image et de spécificité de l'Ecole, la recherche d'intervenants internes et externes de bon niveau.

\subsection{La Commission des titres d'ingénieurs (CTI) et la définition du rôle des SHS}

Sur ce fonds culturel dual, lui-même héritier de longues traditions, la question de la politique d'ensemble des écoles et de leur contrôle pédagogique à une échelle nationale se pose forcément dans un pays centralisé comme la France, et l'on peut prévoir que cela a des incidences sur la question des SHS/Humanités en écoles d'ingénieurs. Depuis l'Entre-deux-guerres (1934), un organisme parapublic autonome, la Commission des titres d'ingénieurs est chargé de suivre ces questions et d'autoriser la délivrance du diplôme et du titre. Les écoles d'ingénieurs sont aujourd'hui soumises à une double évaluation, celle du Ministère de l'enseignement supérieur et de la recherche ou de leur ministère de tutelle quand il est autre, et celle de la CTI, organisme professionnel et pédagogique indépendant seul habilité à valider les cursus d'ingénieurs en France. La CTI agit également par l'édition de documents de références pour la formation des ingénieurs, notamment via son site internet et ses pages « Références et Orientations ${ }^{11}$; certaines concernent les SHS comme nous allons le voir. La CTI réalise d'autre part des missions périodiques d'évaluation, sous forme d'examen des maquettes pédagogiques et de journées passées avec les responsables d'écoles et de départements pour établir ou renouveler les accréditations. Le résultat en est la reconnaissance (ou non) de la formation d'ingénieur candidate pour une durée de temps limitée, assortie de commentaires et de recommandations sur le projet pédagogique. Les exigences de la CTI évoluent au fil du temps, bien entendu, et il peut arriver qu'une formation éprouve des difficultés à être renouvelée, car trop conservatrice et peu sensibles aux nouvelles orientations. Cela peut directement concerner les maquettes de cours de SHS et assimilées, et générer des recommandations aux écoles, comme l'irruption du thème de l'éthique à la fin des années 1990.

Il est intéressant de voir la place attribuée à un domaine élargi des Humanités dans un cadrage général des exigences de la formation des ingénieurs, ou plutôt la définition d'un espace dans lequel peuvent prendre place des enseignements de SHS :

T1.B.1.1.6 - Les compétences essentielles du métier d'ingénieur :

11 Site web de la CTI. 


\section{SHS Web of Conferences}

D'une manière générale la CTI considère que le métier d'ingénieur comporte cinq compétences essentielles :

- la maîtrise des sciences de base et de leur utilisation, socle commun des connaissances et garantie de la rigueur d'analyse et du pouvoir d'adaptation, à long terme, aux exigences évolutives des métiers,

- la maîtrise des sciences de l'ingénieur formant au métier visé, garantes de l'efficacité et du pouvoir d'adaptation, à court terme, du jeune ingénieur,

- la capacité à s'impliquer dans la recherche et à être un vecteur d'innovation,

- l'assimilation de la culture d'entreprise et la compréhension du contexte économique, social, humain, environnemental, éthique, philosophique, ... permettant notamment de s'intégrer dans un groupe et de le diriger efficacement,

- la capacité à communiquer dans un contexte culturel international, sans se limiter à la langue anglaise, ce qui permet l'exercice du métier et de la relation sociale en tout lieu.

(Références et Orientations, t. 1, introduction)

Nous voyons que les deux derniers points, sur cinq, sont explicitement consacrés à des références culturelles et éducatives qui sortent du champ des sciences exactes et de la technologie, et partiellement pour le $3^{\mathrm{e}}$ d'entre eux. En outre, la suite du document indique qu'une enveloppe temps est impartie à cette formation : elle doit atteindre 30\% du temps total passé dans l'Ecole (hors stages). C'est un volume non négligeable, et un vaste espace existe donc au sein des écoles pour envisager des activités de formation aux sciences humaines, sociales, à leurs dimensions opérationnelles mais aussi plus théoriques, aux langues mais aussi à l'inter-culturalité, aux composantes humaines et sociales des projets et des stages des élèves qui préfigurent leur insertion dans le monde professionnel.

C'est un aspect important du rôle joué par la CTI auprès des écoles, durant les trente dernières années, et nombre d'entre elles ont été contraintes d'envisager des programmes de formation aux SHS, de réaliser des recrutements. Cela a surtout joué pour les écoles de spécialités affirmées que nous avons déjà évoquées, à les encourager à dépasser des initiatives jusque-là bien timorées et enkystées dans des traditions de pure efficacité professionnelle. Il faut pour cela rendre hommage à la CTI, lieu de rencontre de directeurs d'écoles de tous types et de représentants de milieux professionnels diversifiés, lieu de débat également. Elle a incontestablement joué un rôle pionnier de réflexion sur les contenus des formations d'ingénieurs et sur leur ouverture au Monde.

Quelles sont les raisons de cette relative importance en termes horaires ? Nous en identifions trois principales. La première réside dans la complexification des données de l'industrie en direction des éléments humains, économiques et sociaux, tant internes qu'externes à l'entreprise.Les relations sociales du travail et l'évolution des hiérarchies, la gestion des ressources humaines en relation avec l'ergonomie et l'organisation de la production, le suivi qualité et sécurité, ont par exemple marqué l'évolution des entreprises ces dernières années ; mais aussi la recherche de la performance individuelle et collective, les contextes pluriels de l'innovation, la diversité des risques sociotechniques, des données plus complexes relatives aux marchés et à la concurrence, la relation client - fournisseur, le marketing et la montée des facteurs technico-commerciaux, etc. Le second élément de cette réussite réside dans la croissance irréversible des données internationales : d'abord l'Europe des trente glorieuses, ensuite la mondialisation, que tout le monde ressent comme stratégique et qui n'est pas sans poser de redoutables questions, non seulement de qualité technique et d'originalité des produits mais aussi d'une bonne approche culturelle des marchés à l'étranger. Enfin, le troisième grand domaine facteur d'évolution est la montée des problèmes de non respect des règles du jeu et des "affaires ». Ils ont joué un rôle, via notamment leur dimension médiatique : propriété industrielle et espionnage industriel, marchés et pots-de-vin, mondialisation financière et blanchiment d'argent, éthique de la production et néoesclavagisme du travail, etc. Nous conviendrons facilement que ces facteurs sont loin d'être seulement technoscientifiques ! 
Ceci dit, les autres consignes qui apparaissent dans les Références et Orientations ont, à mon point de vue, deux orientations générales. La première est de centrer les préoccupations humanistes et sociales sur l'entreprise, et de les ramener d'abord et prioritairement à elle et à son environnement. Nous convenons que cela n'éclaire pas beaucoup sur la nature des SHS à enseigner, sauf à penser qu'il y aurait des SHS spécifiques au monde de l'ingénieur ou, inversement, que les écoles d'ingénieurs sont seulement concernées par des segments bien particuliers des SHS, ceux qui s'intéressent aux métiers de l'ingénieur et à leur exercice dans l'entreprise. Nous pointons ici une difficulté, et peut-être même un malentendu, sur la nature des enseignements à envisager dans la formation des ingénieurs. La seconde est que, même à la lumière des commentaires des Références et Orientation, les indications restent relativement vagues. On se situe bien loin d'une suggestion de programme, que de toute façon la variété des formations concernées rendrait peu opérable. Faut-il s'en plaindre ? Certainement pas, car cela renvoie les acteurs des SHS/Humanités au terrain de la négociation des programmes et des emplois du temps, au cas par cas des écoles voire des départements individuels, une situation que nous abordons dans le second article d'études de cas.

\section{Conclusion}

La question des enseignements non scientifiques et non technologiques en écoles d'ingénieurs est une préoccupation ancienne, qui remonte à leurs origines. Les dénommer aujourd'hui par des labels généraux comme «Humanités » ou «Sciences humaines et sociales » recouvre en fait des situations très diverses, tant au cours de l'histoire des formations techniques en France et à l'étranger, qu'entre une très grande diversité d'écoles aujourd'hui.

Deux grands courants structurent cependant ces formations, à caractère spontanément protéiforme : la tradition de la spécialité technique et celle de l'approche généraliste du métier d'ingénieur. Elles correspondent à des pôles historiquement identifiables et à des traditions de formations, l'une tourné vers l'efficacité professionnelle et une identité bien repérable sur le marché des cadres de l'industrie ou des services, l'autre vers les préoccupations généralistes et socioéconomiques communes aux dirigeants d'entreprises. Nous avons vu que des facteurs externes intervenaient, comme l'action de la CTI en faveur des enseignements autres que scientifiques et techniques. Il en va de même pour la pression du contexte du développement international des entreprises tant dans son aspect d'ouverture culturelle au Monde et à ses marchés que des « affaires » qui en empoisonnent parfois leur bon déroulement.

Les écoles à affichage généraliste se satisfont plutôt bien des exigences intellectuelles et culturelles apportées par les sciences humaines et sociales. Elles ont une bonne compréhension des enjeux larges de la formation pour leurs élèves, comme pour leur image d'école ouverte et dynamique auprès des candidats potentiels. Elles vont parfois jusqu'à promouvoir des domaines originaux de recherche, à l'interface des SPI et des SHS, alimentant par le haut leurs pratiques pédagogiques en la matière ${ }^{12}$. La situation des écoles offrant d'abord une série de spécialités technologiques bien affirmées et de compétences «métiers » ont souvent plus de mal avec ce type d'enseignements, le concevant comme une série de compléments utilitaristes, dont l'objet même n'a souvent que de lointains rapports avec les SHS académiques, dont il faut rappeler que la définition actuelle de leurs champs est récente, plus récente que les écoles d'ingénieurs.

Une série de facteurs a donc promu un espace pédagogique important en direction des sciences humaines et sociales, qui a été rendu obligatoire ou du moins vivement recommandé pour pouvoir officiellement former des ingénieurs en France. Les directives très généralistes qui accompagnent cette obligation laissent finalement toute latitude pour y organiser un peu ce que l'on veut, sous couvert d'un

\footnotetext{
12 Par exemple, dans le domaine de nos propres recherches : Cotte, Michel (éd.)Documents pour l'histoire des techniques : La numérisation du patrimoine technique, éditeur du n ${ }^{\circ} 18$, Paris CNAM, $2^{\mathrm{ème}}$ semestre 2009 ; Cotte, Michel, "Les outils numériques au service de l'histoire des techniques », e-Phaïstos, vol. I-2 Paris, décembre 2012, p. 12-27.
} 


\section{SHS Web of Conferences}

accord de l'école puis de la CTI. Il y a une plage d'initiatives et de développements possibles importante pour les sciences humaines et sociales en écoles d'ingénieurs, mais qu'il faut développer en synergie avec l'ensemble de l'école, c'est-à-dire en jouant pleinement le jeu de ses institutions, de son Conseil des études au niveau conceptuel et de son Service pédagogique en pratique. Le rôle de chacun des partenaires y est décisif, d'un côté la direction de l'école et les responsables des départements technologiques, de l'autre l'équipe SHS qui doit fonctionner comme un véritable collectif avec un projet global et crédible pour l'école. C'est ce que nous examinons dans le second article qu'a bien voulu publier cet ouvrage.

\section{Références}

Barjot, Dominique et Dureuil, Jacques, 2008. 150 ans de génie civil, une histoire de centraliens, D Paris, Presses universitaires Paris-Sorbonne.

Belhoste, Bruno, 1997 «L'alliance entre théorie et pratique », La recherche, n 300, juillet 1997.

Belhoste, Bruno, 1994. Dahan Dalmedico A. et Picon A. (éd.), La Formation polytechnicienne, 1794-1994, Paris, Dunod.

Bergeron, Louis, 1992. Les Hommes d'affaires au temps des Lumières, L'Uomo dell'Illuminismo, Vovelle, Michel, Roma, Ed., Laterza.

Cotte, Michel, 2005. De l'espionnage industriel à la veille technologique, essai sur la circulation des idées techniques durant la révolution industrielle, Belfort - Besançon, UTBM - PU de Franche-Comté.

Cotte, Michel, 2007. Le choix de la révolution industrielle, les entreprises de Marc Seguin et de ses frères (1815-1835), Rennes, PUR Fondation Carnot.

Hervouët, Philippe et Fonteneau, Virginie, 2009. 1919-2009, L'Ecole centrale de Nantes, Nantes, SNER.

Hibbert, Christopher, 1987. The Grand Tour, Londres, Thames Methuen.

Lamard, Pierre et Lequin, Yves-Claude, 2005. La technologie entre à l'Université, Sévenans, UTBM 2005.

Navier, Claude, 1823. Rapport et mémoire sur les ponts suspendus, Paris.

Ott, Florence, 1999. La Société industrielle de Mulhouse (1826-1876), Strasbourg, PU Strasbourg.

Pairault, François, 2000. Gaspard Monge, le fondateur de Polytechnique, Paris, Tallandier.

Picon, Antoine, 1992. L'invention de l'ingénieur moderne, l'Ecole des Ponts et Chaussées, 1747-1851, Paris, Presses de l'ENPC. 